\title{
CAPACIDADES Y DÉFICITS INSTITUCIONALES PARA EL ASOCIATIVISMO INTERMUNICIPAL. LAS MICRORREGIONES COMO RESPUESTA ADAPTATIVA De los Municipios en la ReGión de Nuevo CuYO
}

\author{
Enzo Ricardo Completa (enzocompleta@fcp.uncu.edu.ar) \\ Centro de Investigaciones de la Facultad de Ciencias Políticas y Sociales \\ Universidad Nacional de Cuyo - Argentina
}

\begin{abstract}
En el trabajo de investigación se realiza un análisis exploratorio acerca de las capacidades y déficits institucionales de los gobiernos locales de la Región Nuevo Cuyo para gestionar, de modo asociado con otros municipios, políticas, programas y proyectos a escala microrregional. La investigación pone su foco en el proceso de creación; en las modalidades de gestión y organización que caracterizan al caso en estudio. En función de estos desarrollos, se reflexiona en torno a las restricciones más relevantes en la capacidad institucional que dificultan la ejecución de proyectos asociativos de carácter intermunicipal, lo que permitirá determinar el grado de autonomía que efectivamente detentan los municipios y organismos asociativos municipales en la República Argentina.
\end{abstract}

Palabras clave: Intermunicipal; Microrregion; Capacidad institucional. 


\title{
INSTITUTIONAL CAPACITY AND CONSTRAINTS FOR INTER-MUNICIPAL ASSOCIATIVISM. MICRO-REGIONS AN THE ADAPTIVE RESPONSE FOR MUNICIPALITIES IN THE REGION OF NUEVO CUYO
}

\begin{abstract}
The paper offers an exploratory analysis of the capacities and institutional deficits of local governments in the region of Nuevo Cuyo. We will focus on the way that associated municipalities manage policies, programs and micro-regional projects. The accent will be put on the organization the management of procedures, and more particularly, the legal institutional framework. We shall consider restrictions on institutional capacity to hinder or impede the implementation of partnership projects. We will therefore determine the degree of autonomy actually held by municipalities and associations of municipal agencies in Argentina.
\end{abstract}

Keywords: Micro-regions; Municipalities; Associations. 


\section{INTRODUCCIÓN}

En las últimas décadas los gobiernos locales de la República Argentina experimentaron un intenso proceso de redefinición de sus roles como consecuencia, fundamentalmente, del proceso de descentralización de la administración pública nacional evidenciado durante la década del noventa. Adicionalmente, el proceso de globalización y el desmantelamiento del Estado de Bienestar en Argentina, generaron una nueva distribución de las funciones y competencias entre los diferentes niveles del Estado (nacional, provincial y municipal), aumentando la responsabilidad de los municipios en la gestión del bienestar local.

Como consecuencia de estos procesos, las autoridades locales se vieron obligadas a asumir una multiplicidad de nuevas funciones, competencias y responsabilidades, entre ellas, la generación de políticas públicas inclusivas en materia de educación, seguridad, empleo, pobreza, medioambiente y salud. Todas estas funciones, otrora de exclusiva incumbencia de la nación y de las provincias, se incorporaron a la agenda municipal con posterioridad al desmantelamiento del Estado de Bienestar, sobrecargando las tradicionales competencias locales, vinculadas al mantenimiento del espacio y la infraestructura pública, a la regulación de la industria y el comercio y a la prestación de servicios públicos básicos.

El término desarrollo local traduce con cierta amplitud el conjunto de nuevas funciones asumidas por los municipios en la promoción de sus comunidades, funciones que al presente se ejercen casi con la misma estructura jurídica y atribuciones fiscales que hace unas décadas, lo que ha llevado a un número creciente de autoridades locales a desarrollar nuevas estrategias organizativas y de gestión que les permitan adaptarse a este pronunciado proceso de delegación de funciones y competencias.

La creación de organismos asociativos municipales (microrregiones, consorcios públicos, agencias y asociaciones de desarrollo regional, áreas metropolitanas, etc.) es un fenómeno que surge a partir de la ocurrencia de estos procesos, como una estrategia de gestión innovadora desarrollada por las autoridades municipales para potenciar el crecimiento socioeconómico de las comunidades locales en un contexto competitivo global. 


\section{HACIA UN MODELO DE GESTIÓN HÍBRIDO PARA ORGANISMOS INTERMUNICIPALES}

Dada la situación de inframunicipalismo que caracteriza a la mayoría de las provincias argentinas (Iturburu, 2002; Altschuler, 2003), en donde se observa una gran cantidad de pequeños gobiernos locales sumamente dependientes de niveles superiores de gobierno, con bajos índices poblacionales, estructuras y presupuestos reducidos y escasas capacidades para gestionar las nuevas funciones asumidas tras el proceso descentralizador, resulta relevante analizar las capacidades institucionales de los municipios que participan de experiencias asociativas de carácter horizontal, así como también, de los organismos microrregionales ${ }^{1}$ creados en la Región Nuevo Cuyo².

En este sentido, para llevar adelante una gestión pública intermunicipal a escala microrregional se requiere que las administraciones locales que participen en experiencias asociativas de este tipo cuenten con capacidades institucionales para hacerlo. Definir el tipo de capacidades de gestión que se requieren a tales fines obliga a efectuar algunas aclaraciones conceptuales previas.

En primer término, los problemas que afectan a los municipios argentinos dan cuenta de una serie de falencias en las prácticas administrativas y sistemas de gobierno propios de un modelo de gestión premoderno, con predominio de rasgos patrimonialistas y clientelares. Dicho modelo se encuentra en franca oposición con la gestión racional-burocrática, con la denominada nueva gerencia pública y con el modelo de gestión participativo, que se impusieron como visiones dominantes en diversos ámbitos académicos y políticos de la región durante las últimas dos décadas del siglo pasado, a pesar de que nunca llegaron a implementarse plenamente.

Ahora bien, las nuevas formas de gestión que emergieron de la crisis del Estado de Bienestar pueden, en determinadas circunstancias, complementarse entre sí. Siguiendo este razonamiento, Evans (2003: 2) propone lo que denomina "el

\footnotetext{
1 En el presente artículo se utiliza el término microrregiones para hacer referencia a un tipo específico de organismo cooperativo de carácter intermunicipal (entre municipios exclusivamente) porque el término región se circunscribe exclusivamente a la esfera interprovincial.

2 Creada en el año 1988, la Región Nuevo Cuyo se encuentra integrada por las provincias de Mendoza, San Luis, San Juan y La Rioja.
} 
modelo básico del hibridismo", según el cual se procura alcanzar un equilibrio integrado entre tres modalidades diferentes ( $\mathrm{y}$ a veces contradictorias) de orientar la acción administrativa. De acuerdo al autor, estas modalidades son:

a) La "capacidad burocrática", construida sobre la base del reclutamiento meritocrático, las normas profesionales, la predictibilidad, la carrera funcionaria como recompensa y las estructuras organizacionales coordinadas.

b) Las "señales de mercado", que pueden fortalecer la efectividad de las instituciones públicas en la medida en que alertan sobre la relación costo-beneficio, facilitan una asignación eficiente de los recursos y proporcionan una "disciplina fiscal" para asegurar que las metas establecidas guarden su consistencia en relación con la disponibilidad de recursos.

c) La participación democrática "desde la base", para asegurar que las metas administrativas reflejen las reales necesidades y deseos del ciudadano común, y que los procesos administrativos sean transparentes y responsables.

El "hibridismo" propuesto por Evans, de esta forma, supone la combinación de las capacidades burocráticas weberianas con las capacidades de respuesta del Estado a las señales del mercado y el control democrático ciudadano. Llevado a la esfera municipal del Estado, Claudio Tecco (2008) propone combinar estos diversos modos de gestión de la siguiente forma:

a) La administración burocrática para la realización de determinadas funciones y la prestación de servicios de carácter universal y producción uniforme.

b) La aplicación de lógicas competitivas en áreas en las que sea posible organizar la gestión por resultados.

c) La gestión de redes sociales para atender cuestiones vinculadas al desarrollo económico y social de los territorios.

En un modelo "híbrido" de estas características, las capacidades a desarrollar serán, lógicamente, mayores y más complejas que si se tratase de uno de "tipo puro", sea éste burocrático, gerencial o de redes sociales. Si trasladamos este razonamiento al campo del asociativismo municipal, parece obvio que las capacidades de gestión de los gobiernos locales incidirán en las del organismo microrregional que éstos construyan. 
En el apartado siguiente se precisará el concepto de capacidad institucional en el ámbito de las administraciones municipales, para luego realizar una aproximación al análisis de las principales restricciones y déficits de capacidad institucional para el asociativismo municipal en la Región Nuevo Cuyo, en tanto nueva táctica adaptativa utilizada por un número creciente de municipios en el contexto de las políticas de reforma y modernización del Estado llevadas a cabo luego del retorno de la democracia en el año 1983.

\section{CAPACIDADES Y DÉFICITS INSTITUCIONALES}

El concepto de capacidad institucional comenzó a ser utilizado durante la segunda mitad del siglo pasado en diferentes documentos de estrategia elaborados por organismos internacionales tales como el Banco Interamericano de Desarrollo (BID) y el Programa de Naciones Unidas para el Desarrollo (PNUD). Desde entonces ha formado parte de las discusiones en torno a la necesidad de mejorar la capacidad de las organizaciones públicas, para enfrentar los desafíos del desarrollo de un modo sustentable.

Jorge Hintze (s/f:2) define a la capacidad institucional como "el potencial de las organizaciones para producir valor para terceros o transformarse ellas mismas"; vale decir que, en las organizaciones estatales, el desarrollo de capacidades institucionales está relacionado con la combinación del valor externo y del organizacional necesario para cumplir con la misión y los objetivos principales.

Ahora bien, según Oszlak y Orellana (2000:7) "entre lo que las organizaciones ambicionan hacer y lo que efectivamente consiguen realizar" existen diversos obstáculos que impiden o dificultan la realización de tareas conducentes al logro de los resultados buscados. Estos obstáculos constituyen una brecha a la que Alain Tobelem (1992) denomina "déficit de capacidad institucional" (DCI). De acuerdo a este autor, los DCI más frecuentes pueden agruparse en las siguientes categorías:

- Institucionales propiamente dichos (relacionados con normas, leyes y reglas de juego que condicionan o impiden la ejecución de tareas).

- De coordinación interorganizacional e interjurisdiccional. 
- De organización interna (funciones, estructuras y procedimientos).

- De capacidad financiera y física de la organización.

- De los sistemas de recursos humanos y de remuneraciones.

- De capacidad de los responsables de ejecutar las tareas.

Aun antes de iniciar la investigación, la situación de inframunicipalismo que afecta a la mayoría de los gobiernos locales y la inexistencia de modelos de gestión híbridos lo suficientemente equilibrados y extendidos en el ámbito local argentino nos hace suponer que encontraremos algunos de estos tipos de déficit de capacidad institucional en los municipios que participan de experiencias asociativas en la Región Nuevo Cuyo, así como también, en los organismos microrregionales creados.

\section{ASOCIACIONISMO INTERMUNICIPAL A ESCALA MICRORREGIONAL. LA REGIÓN NUEVO CUYO}

El proceso de descentralización estatal evidenciado en Argentina durante las últimas décadas obligó a los municipios a reformular sus políticas de desarrollo local, a los efectos de hacer frente a la multiplicidad de nuevas funciones y competencias asumidas. En muchos casos, estas funciones exceden el ámbito de resolución territorial de los municipios, así como también sus propias capacidades institucionales para hacerles frente, lo que comenzó a hacer atractiva la generación de políticas cooperativas y asociativas de carácter horizontal.

Una articulación se pone en práctica cuando dos o más organismos acuerdan llevar adelante políticas que se traducen en acciones concretas y en donde cada uno realiza una o más tareas específicas en relación de cooperación horizontal con los otros organismos participantes. La aplicación de políticas asociativas en el ámbito local generalmente desemboca en la creación de un nuevo organismo a cargo de la administración de los proyectos comunes (Cravacuore, Ilari y Villar; 2004: 36). Dentro del arreglo institucional argentino, sobresalen dos tipos de organismos intermunicipales, ambos de creación voluntaria, espontánea y ascendente: 
a) Consorcios públicos: Entidades de carácter asociativo constituidas por dos o más gobiernos locales para la prestación de servicios y la construcción de obras públicas.

b) Microrregiones: A diferencia de los consorcios, suponen un compromiso más grande por parte de los gobiernos locales, quienes se asociaron para construir una instancia de desarrollo mucho más vasta que les permita fortalecer su capacidad institucional y ampliar su poder de negociación frente a terceros.

El éxito de estas iniciativas (en relación a su propagación, desarrollo y vigencia en casi todo el territorio nacional) se debe, entre otras razones, a la sencillez de su proceso de creación, a su estructura organizativa poco compleja, a la posibilidad de modificación y ampliación de sus funciones con facilidad y a la ausencia de restricciones a la hora de disolver los organismos intermunicipales y de incorporar municipios de otras provincias o países vecinos.

De acuerdo a un estudio realizado por el Ministerio del Interior de la Nación, en el año 2002 existían 52 entes intermunicipales en el país (IFAM, 2003). Unos años mas tarde, Daniel Cravacuore y Adriana Clemente (2006) actualizaron este número a 76 , de los cuales el 46\% se encontraba ubicado en la región pampeana, el 18\% en la región patagónica, el 16\% en la región nordeste, el 13\% en la región noroeste y sólo el $7 \%$ en la región cuyana.

Actualmente no contamos con un estudio que dé cuenta de la evolución que tuvo el fenómeno en el país, aunque sí para el caso de la Región Nuevo Cuyo en donde hemos identificado seis (6) microrregiones intermunicipales formalmente constituidas (dos de ellas al presente se encuentran desactivadas) y una microrregión en proceso de conformación ${ }^{3}$, a la cual hemos decidido no incorporar en el análisis por tratarse de un proyecto todavía en ciernes:

3 De acuerdo a la información suministrada por la Directora de Planificación Estratégica de la Municipalidad de Malargüe, Lic. Graciela Viollaz, la Microrregión Cuenca Media del Río Colorado (CUEMECO) estará integrada por los municipios de Malargüe (Provincia de Mendoza), Rincón de las Sauces, Barrancas y Buta Ranquil (Provincia de Neuquén) y tendrá como principal objetivo potenciar el desarrollo económico-productivo de los municipios asociados. 
1. XI Microrregión del Sur Mendocino4.

2. Microrregión Luján de Cuyo-Maipús .

3. Microrregión Intermunicipal Cuyana Andina (MICA) ${ }^{6}$.

4. Microrregión Centro7.

5. Ente Intermunicipal para el Desarrollo de los Municipios del Valle de los Comechingones (ENIDEM) ${ }^{8}$.

\section{Microrregión Ganadera del Noreste de Mendoza ${ }^{9}$.}

En el Cuadro No 1 se compara la población, superficie y densidad demográfica de las microrregiones intermunicipales de la Región Nuevo Cuyo, tomando como base para el análisis los datos correspondientes a los municipios que las integran. Del análisis efectuado se deduce, en primer término, que el rango poblacional de las seis microrregiones es muy variable, oscilando entre 58.800 y 1.800 .000

4 La XI Microrregión del Sur de Mendoza fue creada en junio del año 2000 por los municipios de General Alvear, Malargüe y San Rafael, siendo la primera experiencia de este tipo en la provincia y la onceava del país. A mediados del año 2004 la microrregión fue refundada bajo el nombre "Mendosur".

5 Creada en agosto del año 2000 por los municipios de Maipú y Luján de Cuyo (Provincia de Mendoza) con el fin de avanzar en la solución de diversos problemas comunes y de fomentar la actividad turística y vitivinícola.

6 Fundada en julio de 2004 por los municipios de Las Heras, Lavalle (Provincia de Mendoza) Calingasta y Sarmiento (Provincia de San Juan). En el año 2005 las autoridades de la MICA firmaron un acta de acuerdo complementaria a los efectos de instrumentar la incorporación de la V Región de Chile, compuesta por 38 comunas.

7 La Microrregión Centro fue creada en el año 2005 por los municipios de San Martín, Luján de Cuyo y Maipú de la Provincia de Mendoza con el fin de propiciar acuerdos productivos en el sector del turismo del vino. Ese mismo año, la microrregión consiguió financiamiento para la realización de dos estudios de factibilidad sobre enoturismo financiados por el Ministerio de Economía y Finanzas Públicas de la Nación y el Banco Interamericano de Desarrollo (BID). Desafortunadamente, tras realizarse ambos estudios, los proyectos fueron abandonados. Al presente, la microrregión se encuentra desactivada.

8 Entidad intermunicipal creada a mediados del año 2009 por municipios y comunas de la provincia de Córdoba (Villa Dolores, Conlara, Los Hornillos, Villa Sarmiento, San José, La Paz, San Javier y Yacanto, Los Cerrillos, Las Tapias, Luyaba, Las Rabonas, San Vicente, Sauce Arriba, Villa de las Rosas y La Población), norte de San Luis (Merlo) y sur de La Rioja (Ulapes) con el fin de mancomunar esfuerzos en materia de prestación de servicios, construcción de obras públicas y diseño de estrategias conjuntas de financiamiento.

9 Creada el 13 de marzo de 2001, la Microrregión Ganadera del Noreste de Mendoza tuvo una vida fugaz. Actualmente, los municipios de Lavalle, La Paz y Santa Rosa se encuentran trabajando en su reactivación, propiciando la sanción de un proyecto de ley por el que se instrumente su creación y se garantice su financiamiento. 
habitantes, aproximadamente. La población promedio de los 70 municipios y comunas que componen las microrregiones, por su parte, es de alrededor de 38.578 habitantes, con una alta dispersión que va desde 84 a los 287.000 habitantes.

\section{Cuadro No 1 \\ Microrregiones de la Región Nuevo Cuyo según población, superficie y densidad poblacional}

\begin{tabular}{|c|c|c|c|c|}
\hline \multicolumn{2}{|c|}{ Características demográficas } & Población & Superficie & $\begin{array}{l}\text { Densidad } \\
\text { Poblacional }\end{array}$ \\
\hline \multirow{6}{*}{$\begin{array}{c}\text { Nombre } \\
\text { de la } \\
\text { Microrregión }\end{array}$} & Mendosur & 204.738 hab. & $87.000 \mathrm{~km}^{2}$ & $2,35 \mathrm{hab} . / \mathrm{km}^{2}$ \\
\hline & Luján de Cuyo-Maipú & 258.070 hab. & $5.464 \mathrm{~km}^{2}$ & 47,2 hab. $/ \mathrm{km}^{2}$ \\
\hline & $\begin{array}{l}\text { Ganadera del Noreste } \\
\text { de Mendoza }\end{array}$ & 27.507 hab. & $25.827 \mathrm{~km}^{2}$ & $1,06 \mathrm{hab} . / \mathrm{km}^{2}$ \\
\hline & MICA & 1.784.851 hab. & $60.934 \mathrm{~km}^{2}$ & $29,3 \mathrm{hab} . / \mathrm{km}^{2}$ \\
\hline & Centro & 366.518 hab. & $6.968 \mathrm{~km}^{2}$ & $52,6 \mathrm{hab} . / \mathrm{km}^{2}$ \\
\hline & ENIDEM & 58.805 hab. & * & $*$ \\
\hline
\end{tabular}

Fuente: Elaboración propia en base a datos del Censo Nacional de Población, Hogares y Vivienda de Argentina (2001) y del Censo Nacional de Población, Hogares y Vivienda de Chile (2002).

Este desequilibrio territorial, en términos geográficos, permite inferir las disparidades existentes en el interior de las microrregiones en materia de recursos presupuestarios, desarrollo tecnológico, estructura administrativa y capacidades institucionales, factores estrechamente relacionados con el fenómeno del inframunicipalismo institucional que caracteriza a los municipios de la región.

\section{4. ¿INCAPACIDADES INSTITUCIONALES PARA EL ASOCIACIONISMO MUNICIPAL?}

El análisis de las capacidades institucionales de los municipios de la Región Nuevo Cuyo nos obliga a efectuar algunas aclaraciones previas. En primer término, las circunstancias políticas y socioeconómicas son sumamente distintas en las provincias de la Región Nuevo Cuyo, hecho que condiciona la distribución de competencias entre los distintos niveles de gobierno y, en consecuencia, la configuración de las alianzas y asociaciones entre municipios. 
Tampoco los regímenes municipales revisten características similares en la región. Así, mientras que las constituciones provinciales de San Juan, San Luis y La Rioja han receptado la autonomía municipal en el orden institucional, administrativo, político, económico y financiero, la Constitución de la Provincia de Mendoza no reconoce la autonomía plena a ninguno de sus municipios. De hecho, la Carta Magna mendocina no ha sido reformada desde el año 1916, lo que la convierte en la constitución provincial más antigua del país y en una de las más restrictivas en lo que a su régimen municipal se refiere.

En cuanto al fomento institucional al asociacionismo municipal, solo la Constitución de la Provincia de San Juan habilita expresamente a sus municipios a firmar convenios con otros municipios para la satisfacción de intereses mutuos (art. $251^{\circ}$, inciso 9). El resto de las provincias, por su parte, guardan un silencio absoluto respecto del derecho de asociación municipal, situación que ha llevado a que muchos gobiernos locales no participen en este tipo de iniciativas, prescindiendo de la cooperación intermunicipal incluso cuando se presenta como solución viable a problemas específicos que los municipios no pueden resolver de manera individual.

En cuanto a la normativa de carácter infraconstitucional sancionada en la Región Nuevo Cuyo en materia de cooperación y asociativismo intermunicipal, la misma resulta sumamente escasa y limitada en cuanto a su contenido. En este sentido, solo hemos podido detectarla en la provincia de Mendoza, lo que nos da una idea de las restricciones reales y potenciales que deben afrontar los municipios cuyanos al momento de desarrollar estrategias cooperativas intermunicipales e interinstitucionales.

Respecto del análisis de las funciones, estructuras y procedimientos de las precitadas microrregiones, las mismas han alcanzado diferentes niveles de complejidad y desarrollo organizacional, dependiendo de los distintos contextos políticos y socioeconómicos en los que surgieron y se desenvuelven; del marco jurídico que las regula y de las variaciones que presentan en la cantidad, pertenencia territorial y características organizacionales de los actores involucrados, sin que puedan establecerse correlaciones o constantes entre estas variables, lo que dificulta la comparación de las experiencias asociativas. 
En términos generales, sin embargo, puede afirmarse que el desarrollo organizacional de las microrregiones resulta ciertamente escaso. En este sentido, se evidencian numerosos déficits de coordinación interorganizacional en las mismas, principalmente derivados de la inexistencia de organismos administrativos eficientes que coordinen la gestión intermunicipal y que asignen tareas a los municipios que participan en este tipo de iniciativas; de la escasa presencia de instancias de trabajo, debate y planificación participativa y de la inexistencia de mecanismos para la toma de decisiones y para la resolución de conflictos, tanto en los municipios como en las microrregiones. El bajo desarrollo organizacional de las microrregiones se hace patente, además, en la inexistencia de órganos deliberativos, equipos técnicos de control en las mencionadas instituciones, así como en la ausencia de planes estratégicos microrregionales, reglamentos internos y/o manuales de funciones que aseguren la implementación de los proyectos asociativos.

Adicionalmente, se han detectado numerosos déficits en materia de recursos humanos municipales, entre los que se destacan los siguientes: escasos incentivos para la capacitación del personal y/o para la incorporación de recurso humano calificado en las administraciones municipales; bajo nivel de remuneraciones (hecho que condiciona a los mejores técnicos y operarios a emplearse en el sector privado pero permaneciendo en sus puestos de trabajo en la administración local); reducida capacidad de ejecución de tareas vinculadas a la gestión intermunicipal; insuficiencia de información clave para la toma de decisiones y escaso conocimiento de las autoridades y empleados municipales en materia de gestión pública asociada.

Seguramente la existencia de déficits estructurales en materia de capacidad financiera y física en la mayoría de los municipios que forman parte de las microrregiones se presenta como un grave condicionante para el sostenimiento de este tipo de arreglos institucionales, dificultad que ha llevado a muchos organismos intermunicipales a depender de subsidios provenientes de niveles superiores de gobierno y organismos internacionales de crédito, los cuales son utilizados para contratar personal, para la adquisición de tecnología de información y comunicación (TIC) e incluso para el pago de los gastos corrientes de las microrregiones.

En el futuro, aquellos organismos intermunicipales que no impongan y gestionen sus propias tasas por los servicios prestados (en el caso de que efectivamente se 
eliminen las restricciones institucionales vigentes que impiden la prestación mancomunada de servicios por parte de los organismos intermunicipales) pasarán a depender exclusivamente de las transferencias de recursos que ocasionalmente lleguen desde niveles superiores de gobierno u organismos internacionales de crédito. Por supuesto, la imposición de ciertas tasas intermunicipales no eliminará la necesidad de las aportaciones ordinarias y extraordinarias que deban realizar los municipios para el sostenimiento de estos organismos, e incluso de los subsidios, pero importará un importante avance hacia la autogestión financiera de este tipo de iniciativas.

\section{REFLEXIONES FINALES}

La creación de organismos intermunicipales en Argentina surgió de manera espontánea y ascendente, esto es, sin mediar mandato del gobierno nacional y de las administraciones provinciales, quienes inicialmente vieron con indiferencia $-\mathrm{y}$ en algunos casos, incluso, con disconformidad- ${ }^{10}$ los esfuerzos asociativos de los municipios y comunas, mayormente orientados a disminuir el impacto negativo de los procesos descentralizadores de la administración pública nacional.

Transcurrida una década desde la creación de los primeros organismos intermunicipales en la Región Nuevo Cuyo, se advierte que la falta de políticas públicas y estímulos legales que fortalezcan el reciente proceso de articulación municipal ha atentado contra la institucionalización de estas experiencias, desalentando la conformación de nuevas iniciativas asociativas y haciendo que muchos municipios prescindan de la cooperación y el asociacionismo incluso cuando se presenta como una solución viable para la resolución de problemas regionales.

Respecto de los organismos intermunicipales formalmente creados en la región durante la última década, consideramos que la inexistencia de modelos de gestión híbridos en el ámbito local se encuentra estrechamente relacionada con la ausencia de capacidades institucionales suficientes en los municipios, factor que ha

10 De acuerdo a Cravacuore (2006), las provincias suelen antipatizar con el asociacionismo intermunicipal, dado que éste fomenta los lazos entre los políticos locales, emancipándolos e incrementando su poder de negociación. Inclusive, en algunas provincias, puede encontrarse que procesos de articulación originados en gobiernos locales han sido subsumidos en políticas provinciales. 
condicionado tanto la estructura como el desempeño de los consorcios y microrregiones intermunicipales, imponiendo numerosos impedimentos a la ejecución de tareas que requieren altos niveles de coordinación y complejidad.

Actualmente, la mayoría de las administraciones municipales no mide su propio desempeño en relación con sus capacidades institucionales para el logro de los objetivos planteados. Una situación sumamente preocupante si se tiene en cuenta que la mayoría de los problemas de los ciudadanos se desenvuelven y resuelven (positiva o negativamente) en el ámbito local, lo que convierte al desempeño institucional de los municipios en un factor determinante para dar respuesta a sus deseos y necesidades.

La reducción de los déficits de capacidad institucional mencionados anteriormente constituye un desafío político, técnico y económico que los gobiernos locales de la región deben emprender cuanto antes si desean mejorar el desempeño institucional de los organismos asociativos municipales. El presente trabajo pretende erigirse como un aporte para el desarrollo de prácticas orientadas a la adquisición de nuevas capacidades de gestión municipales en el marco de una agenda local ampliada. En el futuro, esperamos dar continuidad a esta tarea a través de la ejecución de acciones específicas que contribuyan a fortalecer el vínculo entre todos los actores públicos y privados que se entrecruzan en la arena local. 


\section{REFERENCIAS BIBLIOGRÁFICAS}

Altschuler, B. (2003). El asociativismo municipal como estrategia de desarrollo económico en la Argentina. Ponencia presentada en el V Seminario REDMUNI. Mendoza.

Cravacuore, D.; Ilari, S. y Villar, A. (2004). La articulación en la gestión municipal. Actores y políticas. Buenos Aires: Editorial UNQ.

Cravacuore D. y Clemente, A. (2006). El proceso reciente de asociativismo intermunicipal en Argentina. Ponencia presentada en XI Congreso Internacional del CLAD, Guatemala.

Cravacuore, D. (2006). "Análisis del asociativismo intermunicipal en Argentina”. Revista Medio Ambiente y Urbanización. Año 22. No 64, pp. 3-16.

Evans, Peter (2003). "El hibridismo como estrategia administrativa: combinando la capacidad burocrática con las señales del mercado y la democracia deliberativa”. Revista del CLAD. Reforma y Democracia No 25, pp. 1-15.

Hintze; J. (s/f). Guía para la evaluación de la capacidad institucional. Top Herramientas de Gestión. Buenos Aires: Centro de Desarrollo y Asistencia Técnica en Tecnología para la Organización Pública. Disponible en www.top.org.ar

Iturburu, M. (2002). "Nuevos acuerdos institucionales para afrontar el inframunicipalismo argentino", en Mónica Iturburu y Nélida Redondo, Cooperación Intermunicipal en Argentina. Buenos Aires: INAP - EUDEBA.

Oszlak, O. y Orellana, E. (2000). El análisis de la capacidad institucional: aplicación de la metodología SADCI. Documentos TOP sobre Gestión Pública, Buenos Aires: Centro de Desarrollo y Asistencia Técnica en Tecnología para la Organización Pública. Disponible en www.top. org.ar

Tecco, C. (2008). Capacidades de gestión para el desarrollo económico local. Jornadas de Finanzas Municipales. Córdoba, Argentina.

Tobelem, A. (1992). Institutional Capacity Analysis and Development System (ICADS). Operation Manual. World Bank. Public Sector Management Division. Technical Department. Latin American and the Caribbean Region.

El artículo fue recibido el 30 de noviembre y fue aceptado para su publicación el 15 de diciembre. 